\title{
INFRARED-SELECTED “WARM" GALAXIES OBSERVED IN X-RAYS
}

\author{
PAUL J. GREEN ${ }^{1}$, MARTIN WARD ${ }^{2,3}$, SCOTT F. ANDERSON ${ }^{2}$, \\ BRUCE MARGON ${ }^{2}$, M. H. K. DEGRIJP ${ }^{4}$, GEORGE K. MILEY 5 \\ 1. Physics Department, FM-15, University of Washington, Seattle, WA 98195 \\ 2. Astronomy Department, U.WA.; S. IoA,Cambridge \\ 4. Sterrewacht Leiden; 5. STScI, Baltimore
}

\section{Introduction}

The Infrared Astronomical Satellite (IRAS) has detected many galaxies in the infrared (IR), most of which have fairly steep $25 \mu$ to $60 \mu$ spectra. Many quasars and active galaxies exhibit a significantly flatter spectrum in the infrared. Several studies, for example, DeGrijp et al. (1985) used this characteristic to select a subsample of "warm" objects from the IRAS PSC (1985).

We report results of a study which adopts slightly modified IR selection criteria, together with the requirement that the object be observed serendipitously in X-rays by the Einstein Observatory, and not coincide with a bright star on the Palomar Observatory Sky Survey (POSS). Not only is this method complementary to UV, radio, or X-ray samples, but it permits study of correlations between the IR and $X$-rays in a sample with welldefined characteristics in these bands.

\section{Observations \& Selection Criteria}

From the PSC, we selected objects at high galactic latitude $\left(|b| \geq 20^{\circ}\right)$ which are not in the directions of the LMC or the SMC. To examine only "warm" galaxies, we adopt selection criteria similar to DeGrijp et al. (1985) and Kailey and Lebofsky (1988), and require that the spectral index $\alpha$ between 25 and $60 \mu$ be greater (flatter) than -1.5 . This corresponds to a flux ratio $F_{25} / F_{60} \geq 0.645$.

The Einstein Observatory Imaging Proportional Counter (IPC) and High Resolution Imager (HRI) X-ray fields, were sarched for positional overlap within $90^{\prime \prime}$ with the warm objects selected from the PSC. In order to avoid complicating the selection bias of our sample, we reject any objects that were the intended targets of the X-ray fields, since these are presumably weighted toward UV-excess, radio, or optically selected galaxies. X-ray fluxes in the $0.5-3.0 \mathrm{keV}$ range are derived using the background from a $3^{\prime}$ circular annulus around the source.

Visual examination of the POSS eliminates bright galactic objects which otherwise satisfy our criteria. The majority of the objects remaining are galaxies with $B \lesssim 16$. Among those objects for which we have both X-ray information and optical redshifts, the extragalactic sample is at least $62 \%$ complete. Limitations in r.a. and dec, not any 
intrinsic optical selection effect, are responsible for the incompleteness. We obtained lowresolution optical spectra for these objects using the 3.6 meter ESO telescope at La Silla, and the 2.5 meter Isaac Newton telescope at La Palma (De Grijp, Miley and Lub, 1987). Objects with stellar spectra that had not been identified as stars from the POSS were eliminated, leaving an observed sample to date of 16 galaxies.

\section{Conclusions}

Our investigation of IRAS "warm" galaxies has revealed the X-ray detection of a small yet diverse group of AGN. These include a cluster Seyfert 1 (IRAS 04124-0803), a narrow line Seyfert 1 (IRAS 04596-2257), a Seyfert 1.9 (IRAS 14434+2714), a Seyfert 2 (IRAS 12232+1256) and a starburst galaxy (IRAS 02071-1023). Among Seyferts of all types, we detect $44 \%$ of our observed sample. However, we detect only one $(14 \%)$ of the nonSeyfert emission line galaxies. This is consistent with the known fact that Seyfert galaxies have from 100-1000 times higher X-ray luminosities than emission line galaxies. We find a detection rate of $32 \%$ for the entire sample.

There was no a priori X-ray or optical selection criteria applied to these "warm" IRAS galaxies serendipitously observed by Einstein, but because of the small sample size, the X-ray detection percentages should be viewed as indicative. With these qualifications in mind, the percentages may be applied to the much larger complete sample of "warm" IRAS selected galaxies (DeGrijp, Miley and Lub 1987), if they were to be observed with the same distribution of $\mathrm{X}$-ray sensitivities. It is therefore likely that a large number of previously unknown Seyfert 1-1.9s selected by means of their warm IRAS colors could be detected in soft X-rays in future surveys by, for example, ROS AT. A more complete description of this work has been given by Green et al. (1989). This work has been supported by NASA grant NAG8-590.

\section{Further Studies}

In a forthcoming paper, we study correlations between optical, IR and X-ray properties of active galaxies and quasars observed in these three bands. The use of survival analysis substantially enlarges the sample through the inclusion of non-detections in the IR and X-rays. A preliminary result reveals a clear separation between broad line $(B L)$ and narrow line (NL) galaxies in the $\mathrm{L}_{60 \mu}\left(\mathrm{L}_{x}\right)$ relation. At a strong break near $\mathrm{L}_{x}=10^{43} \mathrm{ergs} \mathrm{s}^{-1}$, the IR luminosities of the NL branch and the BL branch differ by a factor of 100 . Seyfert $2 \mathrm{~s}$ may represent a high luminosity extension of the NL phenomenon rather than obscured Seyfert 1s, unless the position of Seyfert $2 \mathrm{~s}$ is due to the conversion of X-rays into IR by the obscuring material.

\section{References}

DeGrijp, M. H. K., Miley, G.K., Lub, J., and de Jong, T. 1985, Nature, \$14, 240.

DeGrijp, M. H. K., Miley, G.K., and Lub, J. 1987, Astr. Ap. Suppl., 70, 95.

Green, P. J. et al. 1989, Ap. J., submitted.

Kailey, W. F., and Lebofsky, M. J. 1988, Ap. J., 326, 653.

IRAS Catalogs and Atlases: Point Source Catalog 1985 (Washington, D. C. : U. S. Government Printing Office). 